\title{
Isolation and molecular characterization of a novel strain of Bacillus with antifungal activity from the sorghum rhizosphere
}

\author{
S.C. Martinez-Absalon ${ }^{1}$, Ma. del C. Orozco-Mosqueda ${ }^{1}$, \\ M.M. Martinez-Pacheco ${ }^{1}$, R. Farias-Rodriguez ${ }^{1}$, M. Govindappa ${ }^{2}$ and \\ G. Santoyo ${ }^{1}$ \\ ${ }^{1}$ Instituto de Investigaciones Quimico-Biologicas, \\ Universidad Michoacana de San Nicolas de Hidalgo, Morelia, \\ Michoacan, Mexico \\ ${ }^{2}$ Department of Biotechnology, Shridevi Institute of Engineering \& Technology, \\ Karnataka, India \\ Corresponding author: G. Santoyo \\ E-mail: gustavo_santoyo@yahoo.com/gsantoyo@umich.mx
}

Genet. Mol. Res. 11 (3): 2665-2673 (2012)

Received January 9, 2012

Accepted May 21, 2012

Published July 10, 2012

DOI http://dx.doi.org/10.4238/2012.July.10.15

\begin{abstract}
We looked for bacterial strains with antifungal activity in the sorghum rhizosphere. A prescreening procedure to search for hemolytic activity among the isolated strains allowed us to detect good fungitoxic activity in a bacterial isolate that we named UM96. This bacterial isolate showed strong growth inhibition in bioassays against the pathogens Diaporthe phaseolorum, Colletotrichum acutatum, Rhizoctonia solani, and Fusarium oxysporum. The supernatant of isolate UM96 also showed strong hemolytic activity, which was not observed in the protease-treated supernatant. However, the supernatant that was treated with protease had similar antagonistic effects to those exhibited by the supernatant that was not treated with this enzyme. These results suggest that a bacteriocin-like compound is responsible for the hemolytic activity; whereas, as far as antifungal effect is concerned, an antibiotic
\end{abstract}


of nonribosomal origin, such as a lipopeptide, might be acting. Further molecular characterization by partial $16 \mathrm{~S}$ rDNA sequencing placed isolate UM96 in a cluster with Bacillus amyloliquefaciens; however, the highest identity match found in databases of Bacillus species was $91 \%$ identity. This suggests that Bacillus sp UM96 might be a novel species.

Key words: Rhizosphere; Hemolytic activity; Fungal pathogens; Bacillus

\section{INTRODUCTION}

The application of agrochemicals to protect crops against diverse fungal plant pathogens is currently occurs worldwide. This practice should be eliminated, however, as the toxic effects of these chemicals on the environment are well documented, as are side effects on human health $(\mathrm{He}$ et al., 2005). An interesting alternative for the control of plagues is the use of bacteria with antagonistic effects against plant pathogens (Weller, 1988; Tomashow, 1996). An important source from which to isolate and select bacteria with biocontrol activities is the rhizosphere of plants (Bloemberg and Lugtenberg, 2001). This microecosystem is defined as the portion of soil influenced by plant roots (Kennedy and de Luna, 2004; Babalola, 2010). The antifungal effect exhibited by various bacterial species against plant pathogens is considered an indirect mechanism for improving plant health. Bacteria with this potential are usually referred to as plant growth-promoting rhizobacteria (Kloepper and Schroth, 1978; Hayat et al., 2010). Thus, the development of new bacterial biocontrol agents is needed to avoid the use of agrochemicals in crops.

Bacterial species belonging to the genus Bacillus have been widely studied and frequently used as biocontrol strains (Leclère et al., 2005; Chen et al., 2009; León et al., 2009). Several Bacillus species produce various antibiotics with antifungal activity, including lipopeptides (Tsuge et al., 2001; Yao et al., 2003; Koumoutsi et al., 2004; Ongena and Jacques, 2008). Lipopeptides such as surfactin, fengycin, and members of the iturin family (e.g., iturin A, bacillomycin D, and mycosubtilin) have been studied in detail and shown to suppress plant pathogenic organisms (Ongena and Jacques, 2008; Chen et al., 2009). Some lipopeptides of the iturin family exhibit specific and strong antagonistic activity against fungi but limited antibacterial action. Some members of this family also show hemolytic activity (Maget-Dana and Peypoux, 1994). Overproduction of mycosubtilin by Bacillus subtilis BBG100 enhances hemolytic activity compared with that of the wild-type strain. This overexpression of the mycosubtilin lipopeptide is also associated with enhanced antifungal and biocontrol activity (Leclère et al., 2005).

In this study we searched for bacterial species with fungitoxic activity in the sorghum rhizosphere. Because hemolytic activity can be associated with antifungal effects and both activities can be present in a single strain, we hypothesized that a pre-screening for hemolytic activity in these isolates to find the desired fungitoxic activities would be faster and require less work. Thus, the bacterial isolate UM96 was selected for its strong hemolytic action and subsequent antagonism against the plant pathogens Diaporthe phaseolorum, Colletotrichum acutatum, Rhizoctonia solani, and Fusarium oxysporum. The activity of bacterial supernatant against fungal pathogens was also evaluated. The $16 \mathrm{~S}$ ribosomal gene sequence from the isolate UM96 was amplified with polymerase chain reaction (PCR) and partially sequenced, showing identity with the Bacillus genus. 


\section{MATERIAL AND METHODS}

\section{Sampling, isolation, and culture conditions}

Sorghum plants (Sorghum spp) with rhizospheric soil were collected from an agricultural field in Morelia, Michoacan, Mexico. To obtain rhizospheric soil, plants were vigorously shaken and soil particles highly attached to the roots were suspended in $100 \mathrm{~mL}$ sterile phosphate buffered saline and shaken. Samples were immediately transported for laboratory analysis. Soil particles (10 tubes with $100 \mathrm{mg}$ each) were separated using centrifugation, and 10-fold serial dilutions of the supernatant were plated on nutrient agar (NA) $\left(\mathrm{BD} \mathrm{DIFCO}^{\mathrm{TM}}\right)$. Agar plates were incubated at $30^{\circ} \mathrm{C}$, and after $48 \mathrm{~h}$ of incubation, more than 300 colonies with various morphologies were chosen for subculture on NA. Isolates were routinely maintained at $4^{\circ} \mathrm{C}$ on NA.

\section{Screening for hemolytic activities}

Hemolytic activities were evaluated by transferring each bacterial colony using sterile toothpicks from NA plates to blood agar plates (BAP) (PROFAM, Mexico). Hemolysis was visualized by the development of a clear hemolytic halo around the colonies after incubation at $30^{\circ} \mathrm{C}$ for $24 \mathrm{~h}$. The probable bacterial colonies surrounded by a halo were chosen. The experiments were repeated in at least triplicate. Isolate UM96 was chosen for further evaluation of hemolytic activities with its supernatant. The supernatant culture was filtered and sterilized with $0.2-\mu \mathrm{m}$ pore size membranes (Millipore). The supernatant was divided into 2 fractions-treated and untreated (2 $\mathrm{h}$ at $37^{\circ} \mathrm{C}$ ) with protease (type XIV; final concentration $10 \mu \mathrm{g} / \mathrm{mL}$ )-and inoculated and spread onto BAP plates $(200 \mu \mathrm{L})$. Hemolytic halos were measured after incubation at $30^{\circ} \mathrm{C}$.

\section{Evaluation of antagonism against plant fungi}

Four plant fungal pathogens were used in this study: D. phaseolorum, C. acutatum, $R$. solani, and F. oxysporum, which were routinely maintained on PDA media at $4^{\circ} \mathrm{C}$. Evaluation of fungal antagonism followed the method described by Filippi et al. (1984). Briefly, the isolate UM96 and pathogenic fungi were simultaneously inoculated on PDA or NA agar plates with identical results. Isolate UM96 was streaked on plates in a cross, and a mycelial plug of $4 \mathrm{~mm}$ was deposited in the center of each of the quadrants. Plates were incubated in darkness at $30^{\circ} \mathrm{C}$, and mycelium growth was measured from day 1 to day 8 .

The culture supernatant of isolate UM96 was also tested for antifungal activities against D. phaseolorum, C. acutatum, R. solani, and F. oxysporum through plate bioassays. Overnight cultures were grown (O.D. 1.2) on NA media, and $2 \mathrm{~mL}$ from dilutions $10^{-0}$ and $10^{-1}$ were inoculated via flooding onto PDA plates and allowed to dry in a laminar flow. A mycelial plug of $4 \mathrm{~mm}$ was deposited in the center of each plate and incubated in darkness at $30^{\circ} \mathrm{C}$. A similar experiment was carried out with overnight cultures, but treatment $\left(2 \mathrm{~h}\right.$ at $\left.37^{\circ} \mathrm{C}\right)$ with protease $(10 \mu \mathrm{g} / \mathrm{mL})$ was added. Mycelium growth was measured at day 8 in both experiments.

\section{Molecular typing of the bacterial isolate UM96}

Genomic DNA was isolated from Bacillus sp UM96 so that the $16 \mathrm{~S}$ ribosomal 
DNA (rDNA) gene could be amplifies using PCR (Sambrook et al., 1982) with the universal bacterial primers Fd1, 5'-CAGAGTTTGATCCTGGCTCAG-3' (forward), and Rd1, 5'-AAGGAGGTGATCCAGCC-3' (reverse), corresponding to positions 8 to 28 and 1526 to 1542 from the Escherichia coli 16S rDNA gene, respectively (Weisburg et al., 1991). The following PCR conditions were used: an initial denaturation at $95^{\circ} \mathrm{C}$ for 3 min followed by 30 cycles of $1 \mathrm{~min}$ at $95^{\circ} \mathrm{C}$ for denaturation, $1 \mathrm{~min}$ at $53^{\circ} \mathrm{C}$ for annealing, and 2 min at $72^{\circ} \mathrm{C}$ for extension, and a final extension step at $72^{\circ} \mathrm{C}$ for $5 \mathrm{~min}$. PCR amplifications were performed with a TC-412 Techne Thermal Cycler. GoTaq ${ }^{\circledR}$ Master Mixes tubes (Promega) were used (tubes are supplied with enzyme, magnesium, deoxyribonucleotide triphosphates, and buffer). Only $0.1 \mu \mathrm{g}$ template DNA and $50 \mathrm{pmoL}$ each primer were added to each tube. The PCR product was additionally purified using a Wizard ${ }^{\circledR}$ SV Gel and PCR Clean-Up System (Promega) following manufacturer instructions. The rDNA $16 \mathrm{~S}$ gene from bacterial isolate UM96 was sequenced at the Laboratorio Nacional para la Diversidad Genomica, CINVESTAV, Irapuato, Mexico. The ribosomal sequence obtained was compared with those deposited in GenBank (National Center for Biotechnology Information) database using the Nucleotide Basic Local Alignment Search Tool program. The nucleotide sequence of the rDNA 16S partial sequence of bacterial isolate UM96 (Bacillus sp) was deposited at the GenBank under accession No. JF430006.

\section{Phylogenetic analysis}

A multiple sequence alignment was generated with ClustalW (www.ebi.ac.uk/ Tools/clustalw2), and the phylogenetic analysis of the 16S rRNA gene sequences was carried out using the Molecular Evolutionary Genetics Analysis 4.0 program (Tamura et al., 2007). The 16S ribosomal sequences of other strains of Bacillus were edited after the alignment to give a similar sequence size $(750 \mathrm{nt})$. To obtain a confidence value for the aligned sequence dataset, we performed a bootstrap analysis of 1000 replications. A phylogenetic tree was constructed using the maximum-parsimony algorithm. Additional methods were also used, and the isolate UM96 was always part of a cluster with Bacillus amyloliquefaciens DSM7.

\section{Statistical analysis}

The relative significance of mycelium growth under various treatments was analyzed using the Student $t$-test $(\mathrm{P}<0.05)$.

\section{RESULTS}

\section{Isolation and identification of hemolytic activity}

Sorghum rhizosphere was sampled to isolate pure bacterial cultures with which to detect hemolytic activity likely associated with antifungal activity. More than 300 isolates were screened for hemolytic characteristics, of which 6 positive strains showed a clear hemolytic halo ( $\beta$-hemolysis). Of these isolates, strain UM96 showed the largest halo (Figure 1). 

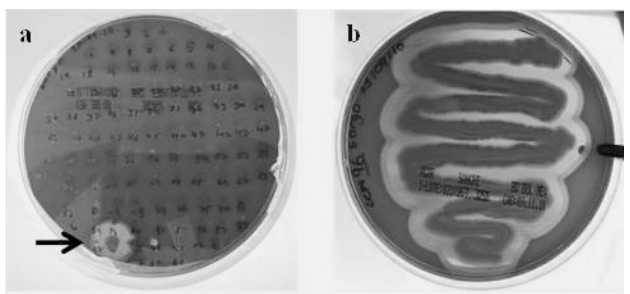

Figure 1. Screening for hemolytic activity among different isolates from sorghum rhizosphere. a. Arrow indicates the hemolytic halo produced by the isolate UM96. b. Isolate UM96 on a blood agar plate showing strong hemolytic activity.

\section{Antagonism against plant pathogens}

Isolate UM96 and 5 other strains were selected after showing strong hemolytic activity on BAP plates (see Figure 1). Interestingly, only isolate UM96 showed robust antagonistic activity against the 4 fungal pathogens tested in plate bioassays, which included $D$. phaseolorum, C. acutatum, R. solani, and F. oxysporum (Figure 2). Both isolate UM96 and the fungi were co-inoculated, and mycelial growth was measured every $24 \mathrm{~h}$ for $6(D$. phaseolorum and $F$. oxysporum $)$ and 8 (C. acutatum and $R$. solani) days. Isolate UM96 significantly $(\mathrm{P} \leq$ 0.05 ) reduced mycelial growth of the 4 fungal pathogens beginning on day 4 by $22 \%$ for $F$. oxysporum, $32 \%$ for $D$. phaseolorum, $35 \%$ for $R$. solani, and $46 \%$ for $C$. acutatum. Thus, the inhibitory effect of isolate UM96 acts in varying degrees with each of the phytopathogens.
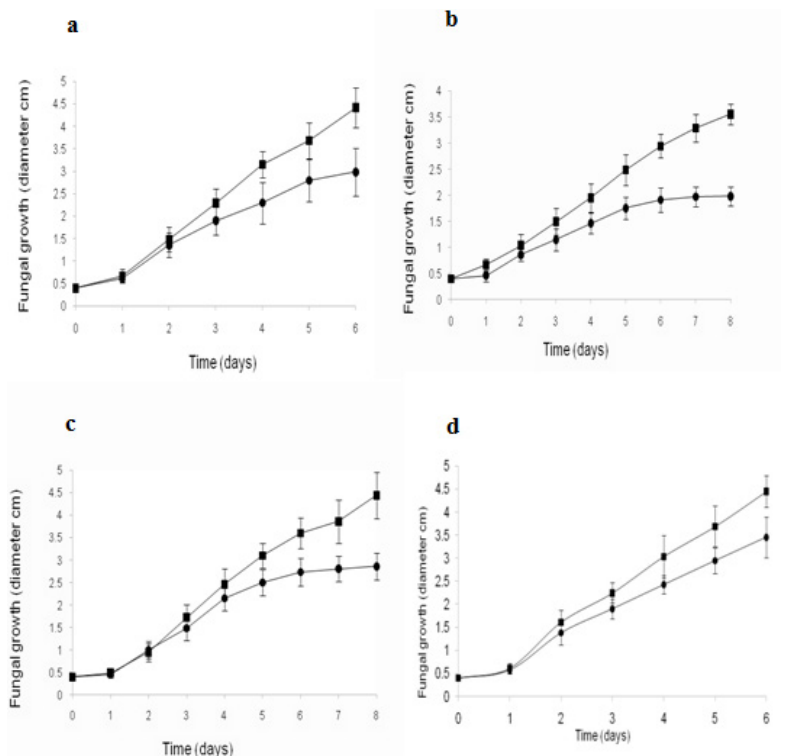

Figure 2. Inhibition of fungal growth by isolate UM96. Fungal growth is presented as the mean of at least four replicates measured in centimeters of diameter, every day for each pathogen, as follows: a) Diaporthe phaseolorum, b) Colletotrichum acutatum, c) Rhizoctonia solani, d) Fusarium oxysporum. Black squares represent pathogen growth in control experiments and black circles represent fungal growth in the presence of isolate UM96. Statistically significant growth inhibition was observed in all pathogens since day $4(\mathrm{P} \leq 0.05)$. 


\section{Hemolytic and antagonistic activities of supernatants}

After observing the hemolytic and antagonistic effects of isolate UM96 against the 4 plant pathogens, we explored the possibility that the compound responsible for this activity could be an antibiotic and diffused into the media. Therefore, supernatant from an overnight liquid culture of the strain was purified and tested for hemolytic activity and inhibitory action against the fungal pathogens. Figure 3 shows the hemolytic halo formed in the supernatant (Figure 3A) and the characteristic hemolysis of strain UM96 (Figure 3B); the protease-treated supernatant showed no hemolytic activity (Figure 3C).

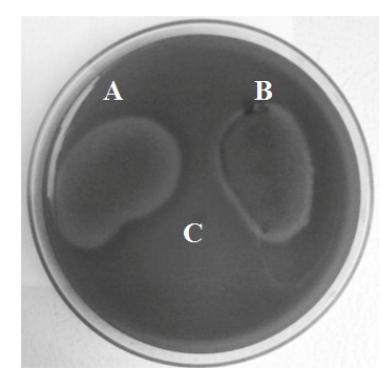

Figure 3. Hemolytic activity of isolate UM96 and its supernatant. Letters show hemolytic halos for supernatant (A) and isolate UM96 (B). Supernatant pretreated with protease did not show hemolysis (C). Experiments were repeated in triplicate, showing representative results.

Once the hemolytic action of the supernatant of isolate UM96 was observed, the possibility of antagonistic effects against the pathogens was also explored. In another experiment, the supernatant was protease treated, eliminating the potential action of bacteriocin-like activity. Table 1 summarizes the results of this experiment. Interestingly, when the supernatant was added directly to the media $\left(10^{-0}\right.$ dilution), the inhibitory effect was significant $(\mathrm{P} \leq 0.05)$ and greater than that observed in previous bioassays. However, when the supernatant was diluted to $10^{-1}$, this effect was reduced by nearly half or less.

Table 1. Antifungal effect of Bacillus sp UM96 supernatant.

\begin{tabular}{|c|c|c|c|c|c|}
\hline \multirow[t]{3}{*}{ Pathogenic fungi } & \multicolumn{5}{|c|}{ Inhibition $(\%)$} \\
\hline & \multirow[t]{2}{*}{$\mathrm{UM}^{\mathrm{a}}{ }^{\mathrm{a}}$} & \multicolumn{2}{|c|}{ Supernatant } & \multicolumn{2}{|c|}{ Supernatant + Protease } \\
\hline & & $10^{-0}$ & $10^{-1}$ & $10^{-0}$ & $10^{-1}$ \\
\hline Diaporthe phaseolorum & $32.39 \pm 9$ & $44.12 \pm 12$ & $14.10 \pm 6$ & $34.22 \pm 11$ & $12.10 \pm 3$ \\
\hline Collectotrichum acutatum & $46.81 \pm 5$ & $68.99 \pm 11$ & $28.92 \pm 8$ & $51.13 \pm 7$ & $27.76 \pm 5$ \\
\hline Rhizoctonia solani & $35.58 \pm 10$ & $52.33 \pm 10$ & $23.78 \pm 9$ & $53.11 \pm 12$ & $31.21 \pm 9$ \\
\hline Fusarium oxysporum & $22.35 \pm 7$ & $48.22 \pm 13$ & $18.35 \pm 4$ & $39.22 \pm 9$ & $16.22 \pm 6$ \\
\hline
\end{tabular}

a Data obtained from Figure 2. Fungal growth inhibition (\%) is reported as means of at least four replicates measured in centimeters of diameter with their respective standard deviation $( \pm)$.

In cases in which the supernatant was protease treated, a slight reduction in inhibitory effect was observed, even when using the $10^{-0}$ dilution. In the case of $R$. solani, no reductive effect was observed in the protease-treated supernatant. These results provide evidence that a probable antibiotic compound in the supernatant from isolate UM96 is responsible for the 
hemolytic activity but not for the antagonism observed against the 4 fungi tested.

\section{Molecular characterization of Bacillus sp UM96}

To characterize isolate UM96 further, the 16S rDNA sequence was isolated and partially sequenced. The highest identity match found in databases for this isolate and other $\mathrm{Ba}$ cillus species was $91 \%$. A phylogenetic tree was also constructed based on a comparison of the 16S rDNA sequences from 9 Bacillus strains (Figure 4). The phylogenetic analysis placed isolate UM96 in a cluster with the bacterium B. amyloliquefaciens DSM7. Although the 16S rDNA gene of UM96 showed the highest identity with B. amyloliquefaciens, more research is necessary to determine the specific taxonomic position of UM96 at the species level.

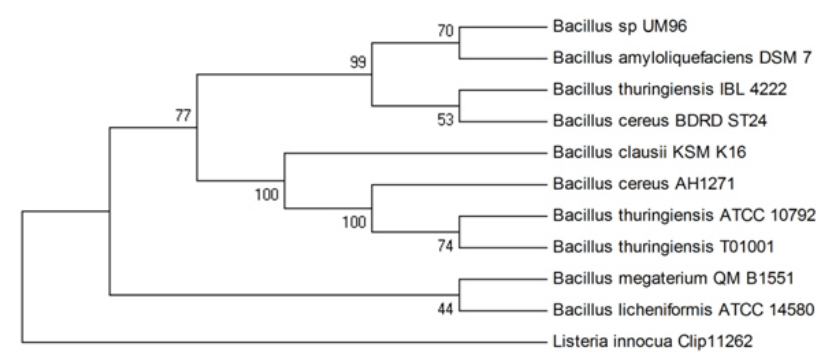

Figure 4. Phylogenetic tree based on $16 \mathrm{~S}$ ribosomal gene sequences (an average of $750 \mathrm{nt}$ ), showing the relationship between Bacillus sp UM96 and other related Bacillus species. The phylogenetic tree was constructed using the maximum-parsimony algorithm. Bootstrap analysis of 1000 replications was performed and is expressed as percentage, and Listeria innocua Clip11262 was used as an outgroup.

\section{DISCUSSION}

Agrochemicals are usually used to control pathogens in crops; however, these xenobiotic compounds have many negative effects on the environment. An alternative option for the biocontrol of pathogens is the use of microorganisms that have no side effects (Bloemberg and Lugtenberg, 2001). Pathogens can be controlled through various mechanisms used by microorganisms (Santoyo et al., 2012). The genus Bacillus contains many species that produce antibiotic compounds with antifungal and biocontrol activities (Ongena and Jacques, 2008). In this study, several bacterial strains were isolated from the sorghum rhizosphere. One isolate, now designated Bacillus sp UM96, showed strong antagonistic action against 4 well-known plant pathogens (D. phaseolorum, C. acutatum, R. solani, and F. oxysporum).

Some bioactive compounds, such as peptides and lipopeptides, are well documented to exhibit hemolytic activity; therefore, a screening strategy was implemented to detect hemolytic activity in the rhizosphere isolates and subsequently search for potential fungal growth inhibitory activity (Koumoutsi et al., 2004; Leclère et al., 2005). Bacillus sp UM96 was the bacterial isolate that showed the clearest and largest hemolytic halo among the hemolytic strains isolated from the sorghum rhizosphere. When Bacillus sp UM96 was tested against various fungal pathogens, it showed robust antagonistic activity, demonstrating a possible association with hemolytic activity, and therefore, the usefulness of the screening. 
This hemolytic and fungitoxic activity was also detected in the supernatant, which displayed hemolytic halos similar to those of the strain on BAP plates. Pretreatment with protease from the supernatants of other hemolytic Bacillus strains resulted in the disappearance of such activity. Protease-sensitive compounds, such as subtilin and subtilosin, are considered responsible for such hemolytic activity (Leclère et al., 2005). Pretreatment with the same protease from the Bacillus sp UM96 supernatant also eliminated hemolytic activity. Thus, similar bacteriocin-like compounds may be responsible for the hemolytic action of strain UM96.

Bacillus sp UM96 supernatants, protease treated or not, were also tested against the fungal pathogens, and the percentages of inhibition were compared to those shown by the strain (see Table 1). Interestingly, greater inhibition percentages were detected in all 4 pathogens when the supernatant was not diluted. When the supernatant was diluted 10-fold, the inhibition zones were reduced by approximately $50 \%$, suggesting that the concentration of the inhibitory compound is important. Interestingly, supernatants pretreated with protease showed only a slight but not significant $(\mathrm{P} \leq 0.05)$ reduction in inhibitory effect on fungal growth. This result strongly suggests that a different bacteriocin-like compound may be responsible for the antagonistic activity.

Bacillus strains not only produce bacteriocins of ribosomal origins but also synthesize other lipopeptides with fungitoxic activity (Ongena and Jacques, 2008)-for example, lipopeptides belonging to the surfactin family (surfactin, lichenysin), the fengycin family (fengycin, plipastatin), and the iturin family (iturin A, C, D, bacillomycin D, F, L, and mycosubtilin). Members of the fengycin family show specific activity against filamentous fungi, whereas some antibiotics of the iturin family also have antifungal (and hemolytic) action (Maget-Dana and Peypoux, 1994). An engineered mycosubtilin-overproducing $B$. subtilis strain, designated BBG100, shows stronger antagonistic and biocontrol activities against different fungal pathogens than those of the wild-type strain (Lecrère et al, 2005). Thus, Bacillus sp UM96 might be producing a nonribosomal lipopeptide involved in antifungal activity. Experiments are currently being carried out to determine the nature of this antibiotic compound (or compounds).

The present study describes a Bacillus sp strain, UM96, isolated from the rhizosphere of sorghum plants that has hemolytic and fungitoxic activities. The partial sequence of the $16 \mathrm{~S}$ ribosomal gene clustered the strain with a type strain of B. amyloliquefaciens DSM7. Several studies have shown that these bacterial species contain gene clusters encoding nonribosomal peptide synthetases and polyketide synthases, which are involved in suppressing plant pathogenic organisms (Koumoutsi et al., 2004; Chen et al., 2009). In addition, B. amyloliquefaciens FZB 45 exhibits plant growth promotion through diverse mechanisms (Idris et al., 2002). Relevant data obtained from the genome sequence of $B$. amyloliquefaciens strain FZB42 indicate that $7.5 \%$ of the whole genome corresponds to genetic elements involved in polyketide and nonribosomal peptide synthetases (Koumoutsi et al., 2004), suggesting genetic characteristics that are relevant to the species.

A phylogenetic analysis revealed that the closest relative of bacterial isolate UM96 is $B$. amyloliquefaciens. The partial $16 \mathrm{~S}$ rDNA sequence contained $91 \%$ identity with that of other $B a-$ cillus species reported in available databases; therefore, bacterial isolate UM96 might be a novel Bacillus species. The accurate taxonomic position of Bacillus sp UM96 at the species level and other relevant roles that it might be playing in plant-bacteria interaction await further research.

\section{ACKNOWLEDGMENTS}

Research supported by the Consejo Nacional de Ciencia y Tecnologia, Mexico (Project 
\#169346) and the Coordinacion de la Investigacion Cientifica of the Universidad Michoacana de San Nicolas de Hidalgo (\#2011-2012).

\section{REFERENCES}

Babalola OO (2010). Beneficial bacteria of agricultural importance. Biotechnol. Lett. 32: 1559-1570.

Bloemberg GV and Lugtenberg BJ (2001). Molecular basis of plant growth promotion and biocontrol by rhizobacteria. Curr. Opin. Plant Biol. 4: 343-350.

Chen XH, Koumoutsi A, Scholz R, Schneider K, et al. (2009). Genome analysis of Bacillus amyloliquefaciens FZB42 reveals its potential for biocontrol of plant pathogens. J. Biotechnol. 140: 27-37.

Filippi C, Bagnoli G, Treggi G and Picci G (1984). Antagonistic effects of soil bacteria on Fusarium oxysporum Schlecht f. sp. Dianthii (Prill and Del.) Snyd. and Hans. I. In vitro experiments and preliminary assays on Carnation (Dianthus caryophyllus L.). Plant Soil 80: 119-125.

Hayat R, Ali S, Amara U, Khalid R, et al. (2010). Soil beneficial bacteria and their role in plant growth promotion: a review. Ann. Microbiol. 60: 579-598.

He ZL, Yang XE and Stoffella PJ (2005). Trace elements in agroecosystems and impacts on the environment. J. Trace Elem. Med. Biol. 19: 125-140.

Idriss EES, Makarewicz O, Farouk A, Rosner K, et al. (2002). Extracellular phytase activity of Bacillus amyloliquefaciens FZB45 contributes to its plant-growth-promoting effect. Microbiology 148: 2097-2109.

Kennedy AC and Luna LZ (2004). Rhizosphere. In: Encyclopedia of Soils in the Environment (Hillel D, ed.). Elsevier Ltd., Oxford, 399-406.

Kloepper JW and Schroth MN (1978). Plant Growth Promoting Rhizobacteria on Radishes. Proceedings of the Fourth International Conference on Plant Pathogen Bacteria (Angers DA, ed.). Vol. 2. INRA, Paris, 879-882.

Koumoutsi A, Chen XH, Henne A, Liesegang H, et al. (2004). Structural and functional characterization of gene clusters directing nonribosomal synthesis of bioactive cyclic lipopeptides in Bacillus amyloliquefaciens strain FZB42. $J$. Bacteriol. 186: 1084-1096.

Leclère V, Bechet M, Adam A, Guez JS, et al. (2005). Mycosubtilin overproduction by Bacillus subtilis BBG100 enhances the organism's antagonistic and biocontrol activities. Appl. Environ. Microbiol. 71: 4577-4584.

León M, Yaryura PM, Montecchia MS, Hernández AI, et al. (2009). Antifungal activity of selected indigenous pseudomonas and bacillus from the soybean rhizosphere. Int. J. Microbiol. Available at [http://dx.doi.org/10.1155/2009/572049] Accessed June 12, 2012.

Maget-Dana R and Peypoux F (1994). Iturins, a special class of pore-forming lipopeptides: biological and physicochemical properties. Toxicology 87: 151-174.

Ongena M and Jacques P (2008). Bacillus lipopeptides: versatile weapons for plant disease biocontrol. Trends Microbiol. 16: $115-125$.

Sambrook J, Frisch EF and Maniatis T (1982). Molecular Cloning: A Laboratory Manual. Cold Spring Harbor Laboratory Press, New York.

Santoyo G, Orozco-Mosqueda Ma del C and Govindappa M (2012). Mechanisms of biocontrol and plant growth-promoting activity in soil bacterial species of Bacillus and Pseudomonas: a review. Biocontrol. Sci. Technol. 22: 855-872.

Tamura K, Dudley J, Nei M and Kumar S (2007). MEGA4: Molecular Evolutionary Genetics Analysis (MEGA) software version 4.0. Mol. Biol. Evol. 24: 1596-1599.

Thomashow LS (1996). Biological control of plant root pathogens. Curr. Opin. Biotechnol. 7: 343-347.

Tsuge K, Akiyama T and Shoda M (2001). Cloning, sequencing, and characterization of the iturin A operon. J. Bacteriol. 183: 6265-6273.

Weisburg WG, Barns SM, Pelletier DA and Lane DJ (1991). 16S ribosomal DNA amplification for phylogenetic study. J. Bacteriol. 173: 697-703.

Weller DM (1988). Biological control of soilborne plant pathogens in the rhizosphere with bacteria. Ann. Rev. Phytopathol. 26: 379-407.

Yao S, Gao X, Fuchsbauer N, Hillen W, et al. (2003). Cloning, sequencing, and characterization of the genetic region relevant to biosynthesis of the lipopeptides iturin A and surfactin in Bacillus subtilis. Curr. Microbiol. 47: 272-277. 